\title{
MOLECULAR SUPERCLOUDS IN M51
}

\author{
T. TOSAKI ${ }^{1}$, R. KAWABE ${ }^{2}$ AND Y. TANIGUCHI ${ }^{1}$ \\ ${ }^{1}$ Astronomical Institute, Tohoku University \\ ${ }^{2}$ Nobeyama Radio Observatory
}

Recently, it has been shown that some of nearby spiral galaxies have very massive $\left(10^{7-8} M_{\odot}\right)$ and large (several $100 \mathrm{pc}-\sim 1 \mathrm{kpc}$ ) molecular gas clouds (M51: Rand \& Kulkarni 1990; NGC1068: Planesas et al. 1991). Since these clouds are significantly more massive and larger than those of so-called Giant Molecular Clouds (GMCs) found in our Galaxy, they are called Molecular Superclouds (Rand \& Kulkarni 1990; hereafter MSCs). In order to study observational properties of MSCs, we present the results of high-resolution $\left(4^{\prime \prime}\right){ }^{12} \mathrm{CO}(\mathrm{J}=1-0)$ mosaic mapping of central 2.'5 region of the Sbc galaxy M51 using the Nobeyama Millimeter Array (NMA). Our main results are summarized in the followings (Tosaki, Kawabe \& Taniguchi 1992).

(1) We have identified $120 \mathrm{MSCs}$ with some reasonable criteria. These MSCs are mainly distributed in the spiral arms and are associated with the giant HII regions.

(2) The typical mass and size of MSCs are $\sim 10^{7} M_{\odot}$ and several $100 \mathrm{pc}$, respectively. Some MSCs tend to build up larger association whose masses and sizes amount to $10^{8} M_{\odot}$ and $1 \mathrm{kpc}$, respectively. They correspond to Giant Molecular Associations (GMAs) studied by Rand \& Kulkarni (1990)

(3) The mass-size relation for MSCs is well described as a power-law form, $M(D) \propto D^{2.1 \pm 0.1}$. This mass-size relation for MSCs is mimic to that for GMCs, molecular cores, and dark clouds in our Galaxy. Although it is possible to interpret this relation for MSCs by an idea that the ${ }^{12} \mathrm{CO}$ emission is saturated, we may explain mass-size relation of MSCs using the turbulence models in analogy to that of molecular clouds in our Galaxy. If this is the case, it is suggested that the universality of mass-size relation are extended even to the class of MSCs.

(4) The mass function of MSCs is well described as a power-law form with a power index of -1.5 . This value is similar to that for the GMCs in our Galaxy (-1.6: Scoville \& Sanders 1986).

(5) The origin of MSCs is briefly discussed. As first, it is unlikely that the MSCs are formed from GMCs by collisional agglomeration, because the collision time scale for GMCs $\left(\tau_{\text {coll }}^{\text {(GMC) }} \sim 9.4-27 \times 10^{7}\right.$ years $)$ is longer than lifetime of MSCs ( $\tau_{\text {MSC }} \sim 5 \times 10^{7}$ years). However, for smaller clouds than GMCs with mass of $500 M_{\odot}$, it is possible that MSCs are formed by collisional agglomeration of 


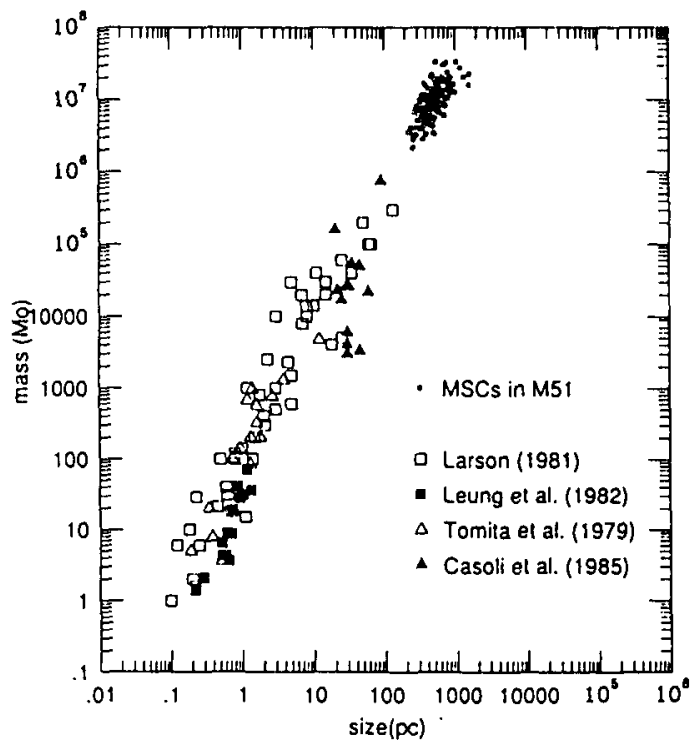

FIGURE I A comparison of the mass - size relations between MSCs in M51 and molecular clouds in our Galaxy. The data of molecular clouds in our Galaxy are taken from Larson (1981), Leung it et al. (1982), Tomita et al. (1979), and Casoli et al. (1983).

small clouds because of the shorter collision timescale for such small molecular clouds $\left(\tau_{\text {coll }} \sim 4.5-13 \times 10^{7}\right.$ years $)$. Secondly, it is possible that MSCs are formed through fragmentation by gravitational instability in a gas disk because the surface density of molecular gas in M51 is high, $55 M_{\odot} \mathrm{pc}^{-2}$, enough to cause the instability.

\section{REFERENCES}

Casoli, F., Combes, F., \& Gerin, M. 1984, $A \& A$, 133, 99

Larson, R. B. 1981, MNRAS, 194, 809

Leung, C. M., Kutner, M. L., Mead, K. N. 1982, $A p J, 262,583$

Planesas, P., Scoville, N. Z., \& Myers, S. T. 1991, ApJ, 369, 364

Rand, R. J., \& Kulkarni, S. R. 1990, ApJ, 349, L43

Scoville, N. Z., \& Sanders, D. B. 1987, in "Interstellar Processes", eds. D. J. Hollenbach, H. A. Thronson, Jr., Reidel, p. 21

Tomita, Y., Saito, T., Ohtani, H. 1979, PASJ, 31, 407

Tosaki, T., Kawabe, R. \& Taniguchi, Y. 1992, in preparation 